\title{
Efmoroctocog Alfa: A Review in Haemophilia A
}

\author{
James E. Frampton ${ }^{1}$ \\ Accepted: 22 September 2021 / Published online: 7 November 2021 \\ (c) Springer Nature 2021, corrected publication 2021
}

\begin{abstract}
Efmoroctocog alfa (Elocta ${ }^{\circledR}$, Eloctate $^{\circledR}$, Eloctate $\left.{ }^{\mathrm{TM}}\right)$, an extended half-life (EHL) recombinant factor VIII (rFVIII)-Fc fusion protein, is approved for the treatment and prophylaxis of bleeding in patients with haemophilia A. The efficacy of efmoroctocog alfa in the prevention and treatment of bleeding in previously treated patients (PTPs) and previously untreated patients (PUPs) with severe haemophilia A has been demonstrated in phase III studies; this includes its use in the perioperative setting (in PTPs). Furthermore, the effectiveness of efmoroctocog alfa in clinical practice has been confirmed in numerous real-world studies; compared with conventional, standard half-life (SHL) FVIII products, prophylaxis with this EHL FVIII product achieved similar or reduced bleeding rates with fewer injections. Efmoroctocog alfa was generally well tolerated; inhibitors occurred in approximately one-third of PUPs in a phase III study. Efmoroctocog alfa is an established and effective EHL FVIII replacement therapy for the management of haemophilia A. Compared with SHL FVIII products, EHL FVIII products such as efmoroctocog alfa have the potential to optimise prophylactic outcomes by decreasing the burden of treatment or increasing the level of bleed protection.
\end{abstract}

\section{Plain Language Summary}

Coagulation factor VIII (FVIII) replacement therapy is the mainstay of haemophilia A treatment; FVIII prophylaxis is the standard of care for severe disease. EHL rFVIII products have been developed to decrease the burden and/or increase the effectiveness of prophylaxis compared with conventional FVIII/rFVIII products which, due to their shorter half-lives, require more frequent injections. Efmoroctocog alfa $\left(\right.$ Elocta $^{\circledR}$, Eloctate $^{\circledR}$, Eloctate $\left.{ }^{\mathrm{TM}}\right)$, a first-in-class rFVIII-Fc fusion protein with a half-life $\approx 1.4-1.8$ times longer than that of conventional FVIII/rFVIII preparations, is approved for the prophylaxis and treatment of bleeding in patients with haemophilia A in various countries worldwide. The efficacy of efmoroctocog alfa has been demonstrated in phase III trials in patients with severe haemophilia A, and its effectiveness, particularly as FVIII prophylaxis, has been confirmed in numerous studies in clinical practice. The rate of formation of neutralizing anti-FVIII antibodies (inhibitors) with efmoroctocog alfa is similar to that with other FVIII/rFVIII products. Based on a large body of clinical trial and real-world data, efmoroctocog alfa is an established and effective EHL FVIII replacement therapy for the management of haemophilia A.

Digital Features for this Adis Drug Evaluation can be found at https://doi.org/10.6084/m9.figshare.16728433.

The manuscript was reviewed by: J.N. Mahlangu, Haemophilia Comprehensive Care Centre, School of Pathology, Faculty of Health Sciences, University of the Witwatersrand and National Health Laboratory Service, Johannesburg, South Africa; D.V. Quon, Orthopaedic Hemophilia Treatment Center, Los Angeles, CA, USA; M. Schiavoni, Associazione per la Lotta alle Malattie Emorragiche e Trombotiche, Maglie, Italy.

James E. Frampton

demail@springer.com

1 Springer Nature, Private Bag 65901, Mairangi Bay, Auckland 0754, New Zealand

\section{Introduction}

Coagulation factor VIII (FVIII) replacement therapy via intravenous (IV) administration of plasma-derived FVIII or recombinant FVIII (rFVIII) concentrates is still the mainstay of treatment for haemophilia A [1-3]. For individuals with severe disease [endogenous FVIII activity $<1 \mathrm{IU} / \mathrm{kg}$ ( $<1 \%$ of normal; $\approx 50-60 \%$ of patients)], optimal treatment would include prophylactic therapy aimed at preventing bleeding, particularly haemarthroses; prophylaxis initiated in early childhood is effective in preserving joint health [1, $2,4]$. Prophylaxis also reduces the formation of neutralizing 
Efmoroctocog Alfa: Clinical Considerations in Haemophilia A

First-in-class rFVIII-Fc fusion protein.

Half-life $\approx 1.4-1.8$ times that of conventional rFVIII products.

Efficacy and safety demonstrated in phase III clinical trials; effectiveness demonstrated in real-world studies.

Expected rate of inhibitor development in PUPs.

anti-FVIII antibodies (inhibitors), which is the most serious complication of haemophilia treatment [5].

Traditional FVIII products (including conventional rFVIII preparations) have relatively short half-lives $(\approx 10-14 \mathrm{~h})$ that necessitate frequent injections (typically three or more times a week) to maintain protective FVIII levels ( $>1 \mathrm{IU} / \mathrm{kg}$ ). Thus, rFVIII preparations with extended half-lives have been developed, which have the potential to optimise prophylactic outcomes by (i) decreasing the burden of treatment through a reduction in injection frequency [which may promote adherence and improve patients' health-related quality of life (HRQOL)] or (ii) increasing the FVIII trough level (and hence the amount of bleed protection) without reducing injection frequency [1-4].

Efmoroctocog alfa $\left(\right.$ Elocta $^{\circledR}$, Eloctate $^{\circledR}$, Eloctate $\left.{ }^{\mathrm{TM}}\right)$, a first-in-class rFVIII-Fc fusion protein consisting of a single molecule of recombinant B-domain deleted human FVIII covalently linked to the dimeric Fc domain of human immunoglobulin G1, is one such extended half-life (EHL) rFVIII preparation $[2,5]$. It is approved for the treatment and prophylaxis of bleeding in patients with haemophilia $\mathrm{A}$ in various countries worldwide, including those of the EU [6], as well as the USA [7] and Japan [8]. This article discusses clinical trial and real-world data on the use of efmoroctocog alfa in the treatment and prophylaxis of bleeding episodes in patients with haemophilia A.

\section{Pharmacological Properties}

The pharmacological properties of efmoroctocog alfa, including preclinical studies evaluating the immunomodulatory properties of this and other Fc fusion proteins in models of haemophilia A, have been reviewed in detail elsewhere [2, 9].

Key pharmacokinetic parameters for efmoroctocog alfa are summarized in Table 1. The pharmacokinetics of this EHL rFVIII product are age-dependent, with clearance decreasing and, therefore, the terminal elimination half-life $\left(t^{1} / 2\right)$ increasing, with increasing age [2] (Table 1). The $t^{1 / 2}$ of efmoroctocog alfa is $\approx 1.4-1.8$ times longer than that of conventional FVIII/rFVIII preparations as a result of the Fc portion binding to the neonatal Fc receptor, which is part of a naturally occurring pathway that protects immunoglobulins (and Fc fusion proteins) from lysosomal degradation by recycling them back into the circulation $[2,5,10]$.

\section{Therapeutic Efficacy of Efmoroctocog Alfa}

\subsection{Previously Treated Patients}

The efficacy of efmoroctocog alfa in the prevention and treatment of bleeding episodes in previously treated patients (PTPs) with severe haemophilia A has been demonstrated in two open-label, noncomparative, multinational, phase III trials (A-LONG [11] and Kids A-LONG [12]) and confirmed in a long-term extension study (ASPIRE) [13].

Table 1 Pharmacokinetic parameters of efmoroctocog alfa: results by age group in previously treated males with severe haemophilia A who received a single intravenous dose (50 IU/kg) in phase III studies [6]

\begin{tabular}{|c|c|c|c|c|c|c|c|c|}
\hline \multirow[t]{3}{*}{ Parameter (geometric mean) } & \multicolumn{4}{|c|}{ Kids A-LONG } & \multicolumn{4}{|l|}{ A-LONG } \\
\hline & \multicolumn{2}{|l|}{$<6$ years } & \multicolumn{2}{|c|}{$6-11$ years } & \multicolumn{2}{|c|}{$12-17$ years } & \multicolumn{2}{|c|}{$\geq 15$ years } \\
\hline & $\begin{array}{l}\text { OSCA } \\
(n=23)\end{array}$ & $\begin{array}{l}\mathrm{CA} \\
(n=24)\end{array}$ & $\begin{array}{l}\text { OSCA } \\
(n=31)\end{array}$ & $\begin{array}{l}\mathrm{CA} \\
(n=27)\end{array}$ & $\begin{array}{l}\text { OSCA } \\
(n=11)\end{array}$ & $\begin{array}{l}\mathrm{CA} \\
(n=11)\end{array}$ & $\begin{array}{l}\text { OSCA } \\
(n=28)\end{array}$ & $\begin{array}{l}\text { CA } \\
(n=27)\end{array}$ \\
\hline IR (IU/dL per IU/kg) & 1.90 & 1.88 & 2.30 & 2.08 & 1.81 & 1.91 & 2.24 & 2.49 \\
\hline AUC/dose (IU.h/dL per IU/kg) & 28.9 & 25.9 & 38.4 & 32.8 & 38.2 & 40.8 & 51.2 & 47.5 \\
\hline $\mathrm{CL}(\mathrm{mL} / \mathrm{h} / \mathrm{kg})$ & 3.46 & 3.86 & 2.61 & 3.05 & 2.62 & 2.45 & 1.95 & 2.11 \\
\hline$t_{1 / 2}(h)$ & 12.3 & 14.3 & 13.5 & 15.9 & 16.0 & 17.5 & 19.0 & 20.9 \\
\hline MRT (h) & 16.8 & 17.2 & 19.0 & 20.7 & 22.7 & 23.5 & 25.2 & 25.0 \\
\hline $\mathrm{V}_{\mathrm{ss}}(\mathrm{mL} / \mathrm{kg})$ & 57.9 & 66.5 & 49.5 & 63.1 & 59.4 & 57.6 & 49.1 & 52.6 \\
\hline
\end{tabular}

$A U C$ area under the factor VIII activity-time curve, $C A$ chromogenic assay, $C L$ clearance, $I R$ incremental recovery, $I U$ international unit, $M R T$ mean residence time, $O S C A$ one-stage clotting assay, $t_{1 / 2}$ terminal elimination half-life, $V_{s s}$ volume of distribution at steady state 
Previously treated adult and adolescent males aged $\geq 12$ years with $<1 \%$ endogenous FVIII activity (or severe genotype) were eligible to enter A-LONG if (i) they were being treated prophylactically or (ii) they were being treated episodically and had a history of $\geq 12$ bleeding events in the 12 months prior to the study. This partially randomized trial had three arms: individualized prophylaxis (IP) $[25 \mathrm{IU} / \mathrm{kg}$ on day 1 and $50 \mathrm{IU} / \mathrm{kg}$ on day 4 to start; thereafter, dose (25-65 IU/kg) and dosing interval (every 3-5 days) adjusted as needed]; weekly prophylaxis (WP) [65 IU/kg]; and episodic treatment (ET) [10-50 IU/kg, depending on bleeding severity] [11]. Co-primary efficacy endpoints included the overall per-patient annualized bleeding rate (ABR) in the IP arm versus the ET arm and basic pharmacokinetic parameters for efmoroctocog alfa [11] (Table 2).

Children aged $<12$ years with $<1 \%$ endogenous FVIII activity (or severe genotype) were eligible to enter Kids A-LONG if they had been treated with any FVIII product for $\geq 50$ exposure days (EDs) [12]. All 69 evaluable patients (35 aged $<6$ years; 34 aged 6-11 years) in this single-arm, non-randomized study received IP [a twice-weekly regimen of $25 \mathrm{IU} / \mathrm{kg}$ on day 1 and $50 \mathrm{IU} / \mathrm{kg}$ on day 4 to start; thereafter, dose ( $\leq 80 \mathrm{IU} / \mathrm{kg}$ ) and dosing interval ( $\geq 2$ days) were adjusted as needed]. The sole primary endpoint was the development of inhibitors detected at $\geq 0.6$ Bethesda units (BU)/mL [12] (Sect. 4).

Patients who completed A-LONG or Kids A-LONG could enrol in ASPIRE [13]. This non-randomized, open-label, multinational, phase III trial had four treatment groups: (1) IP [25-65 IU/kg every 3-5 days or twice-weekly dosing with 25-65 IU/kg on day 1 and 40-65 IU/kg on day 4 (doses $\leq 80 \mathrm{IU} / \mathrm{kg}$ with dosing intervals $\geq 2$ days in patients aged $<12$ years)], (2) WP (65 IU/kg), (3) modified prophylaxis (MP) [personalized dosing for patients in whom optimal prophylaxis could not be achieved with either IP or WP, e.g. less frequent dosing or targeting a FVIII trough level of $>3 \%$ ] and (4) ET (dosing based on type and severity of bleeding). Patients could change from one treatment group to another at enrolment (and at any time during the trial), except those aged $<12$ years at enrolment who could only participate in the IP and MP groups until the age of 12 . The primary endpoint was the development of inhibitors (Sect. 4); secondary endpoints included bleed control (e.g. ABRs and resolution of acute bleeding episodes) [13].

\subsubsection{Prophylaxis}

Long-term prophylactic treatment with efmoroctocog alfa (administered once- or twice-weekly in adults/adolescents; twice-weekly in children) was effective in preventing bleeding episodes in male PTPs with severe haemophilia, including those with pre-existing target joints (Tables 2, 3).

The annualized bleeding rate (ABR) in A-LONG (primary objective) was significantly reduced by $92 \%$ with IP versus ET (2.9 vs. $37.3 ; p<0.001)$ and by $76 \%$ with efmoroctocog alfa as WP versus ET (8.9 vs. 37.3; $p<0.001$ ). Reductions in ABRs with both IP and WP relative to ET were consistent across all prespecified subgroups [11].

The overall median ABR in the efmoroctocog alfa IP arm in A-LONG was 1.6 (Table 2); the last 6 months onstudy median ABR in 23 patients with $\geq 9$ months on study was zero [14]. The median dosing interval was 3.5 days

Table 2 Efficacy of efmoroctocog alfa in the prophylaxis of bleeding episodes in phase III studies of previously treated males with severe haemophilia A

\begin{tabular}{|c|c|c|c|c|c|c|}
\hline \multirow[t]{2}{*}{ Outcome } & \multicolumn{3}{|c|}{ A-LONG (pts $\geq 12$ y) $[2,6,11]$} & \multicolumn{3}{|c|}{ Kids A-LONG ${ }^{\mathrm{a}}$ (pts $<12$ y) $[2,7,12]$} \\
\hline & $\mathrm{IP}(n=117)$ & $\mathrm{WP}(n=23)$ & $\mathrm{ET}(n=23)$ & All pts $(n=69)$ & $<6$ y $(n=35)$ & $6-11$ y $(n=34)$ \\
\hline Median treatment duration (wks) & 32.1 & 28.0 & 28.9 & 26.3 & NR/NA & NR/NA \\
\hline \multicolumn{7}{|l|}{ Median ABR } \\
\hline Overall & 1.6 & 3.6 & 33.6 & 2.0 & 0.0 & 2.0 \\
\hline Spontaneous & 0.0 & 1.9 & 20.2 & 0.0 & 0.0 & 0.0 \\
\hline Traumatic & 0.0 & 1.7 & 9.3 & 0.0 & 0.0 & 0.0 \\
\hline Joint & 0.0 & 1.9 & 22.8 & 0.0 & 0.0 & 0.0 \\
\hline Spontaneous & 0.0 & 0.0 & 18.6 & 0.0 & 0.0 & 0.0 \\
\hline Traumatic & 0.0 & 0.0 & 3.9 & NR/NA & NR/NA & NR/NA \\
\hline Pts with 0 bleeds (\%) & 45.3 & 17.4 & 0 & 46.4 & 51.4 & 41.2 \\
\hline Median dosing interval (d) & 3.5 & 7 & NR/NA & 3.5 & 3.5 & 3.5 \\
\hline Median CFC/wk (IU/kg) & 77.9 & 65.6 & NR/NA & 88.1 & 91.6 & 86.9 \\
\hline Median CFC/y (IU/kg) & 4212 & 3805 & 1039 & NR/NA & 5146 & 4700 \\
\hline
\end{tabular}

$A B R$ annualized bleeding rate, $C F C$ clotting factor consumption, $d$ days, $E T$ episodic (on-demand) treatment, $I P$ individualized prophylaxis, $I U$ international unit, $N R / N A$ not reported or not available/applicable, $p t s$ patients, $w k(s)$ week(s), $W P$ weekly prophylaxis, $y$ year

${ }^{a}$ All pts received IP with efmoroctocog alfa 
(Table 2). Of note, patients transitioning from pre-study prophylaxis with conventional FVIII to efmoroctocog alfa IP experienced reductions in both ABR and injection frequency [15]. Thus, the overall and last 3 months on-study median ABRs (2.1 and 0.0, respectively) were significantly $(p<0.001)$ lower than the pre-study median ABR in patients previously on prophylactic therapy who received efmoroctocog alfa IP for $\geq 6$ months $(n=80)$. All but one of these patients decreased their injection frequency compared with their pre-study regimen, albeit their median weekly FVIII consumption remained consistent [15].

Similar to the efmoroctocog alfa IP arm of A-LONG, the median overall ABR in Kids A-LONG was 2.0 (Table 2); the last 3 months on-study median ABRs in 26 patients aged $<6$ years and 33 patients aged $6-11$ years with $\geq 24$ weeks on study were zero and zero, respectively [12]. The median dosing interval was 3.5 days (in both age cohorts) (Table 2). Patients who had previously received FVIII prophylaxis showed a lowering of their overall median ABR [pre- vs onstudy: 1.5 vs 0.0 in the $<6$ year cohort $(n=32) ; 2.5$ vs 2.0 in the $6-11$ year cohort $(n=30)$ ]. Moreover, $74 \%$ reduced their injection frequency compared with their pre-study regimen; their median weekly FVIII consumption was also lower (prevs on-study: 97.8 vs 90.6 and 102.5 vs $87.7 \mathrm{IU} / \mathrm{kg}$ in the $<6$ and 6-11 year cohorts, respectively) [12].

A-LONG participants receiving efmoroctocog alfa IP reported clinically meaningful improvements in HRQOL, as assessed using the Haemophilia-specific Quality of Life (Haem-A-QoL) questionnaire, an instrument which has been designed and validated for use in adults (i.e. $\geq 17$ years of age) with haemophilia [16]. At 6 months, improvements from baseline in the Haem-A-QoL 'total score' and the 'physical health' domain score were significant $(p \leq 0.034)$ in evaluable patients who received IP. Improvements in HRQOL were more marked and responder rates were higher in patients who received pre-study ET with FVIII than those who received pre-study prophylactic treatment with FVIII [16].

A-LONG participants receiving efmoroctocog alfa IP also experienced some significant improvements in pain-related QOL over time (post hoc data) [17]. As assessed using the Haem-A-QoL 'physical health' domain, the proportions of evaluable patients who reported that they never/rarely experienced painful swellings ( $66 \%$ vs $46 \% ; p=0.001)$ and never/rarely experienced pain in their joints $(42 \%$ vs $27 \% ; p=0.012$ ) were greater at the end of the study than at baseline. In addition, as assessed using the "pain/discomfort' domain of the 3-level version of the EuroQol 5-Dimension (EQ-5D-3L) instrument], the proportion of evaluable patients who reported no pain/discomfort was greater at the end of the study than at baseline (45\% vs $34 \%$; $p<0.05)$. Mean changes from baseline in EQ-5D-3L index and visual analogue scale scores were significant $(p<0.05)$ at the end of the study [17].

Of note, the majority of patients treated with efmoroctocog alfa in A-LONG or Kids A-LONG (86\% and 87\%, respectively) reported maintained or increased physical activity levels during the period of assessment (weeks 7-52 and 2-26, respectively) [18]. Similar results were seen in patients with and without target joint(s) at baseline [18]

Table 3 Efficacy of efmoroctocog alfa in the long-term prophylaxis of bleeding episodes in the phase III ASPIRE study in previously treated males with severe haemophilia A: results by core study and treatment regimen $[6,13]$

\begin{tabular}{|c|c|c|c|c|c|c|}
\hline \multirow[t]{2}{*}{ Outcome } & \multicolumn{4}{|c|}{ A-LONG [pts $\geq 12 \mathrm{y}](n=150)^{\mathrm{a}}$} & \multicolumn{2}{|c|}{ Kids A-LONG [pts $<12$ y] $(n=61)^{\mathrm{a}, \mathrm{b}}$} \\
\hline & $\mathrm{IP}(n=110)$ & $\mathrm{WP}(n=27)$ & $\operatorname{MP}(n=21)^{\mathrm{c}}$ & $\mathrm{ET}(n=13)$ & IP $[<6$ y $](n=29)$ & $\operatorname{IP}[6-11 \mathrm{y}](n=30)$ \\
\hline \multicolumn{7}{|l|}{ Median ABR } \\
\hline Overall & 0.7 & 2.2 & 4.1 & 19.1 & 1.2 & 1.6 \\
\hline In pts with $\geq 1$ target joint $^{\mathrm{d}}$ & 0.7 & 2.2 & 5.0 & 16.1 & 5.0 & 0.8 \\
\hline Spontaneous & 0.1 & 1.5 & 1.4 & 14.6 & 0.6 & 0.3 \\
\hline Traumatic & 0.2 & 0.5 & 0.9 & 1.4 & 0.4 & 1.0 \\
\hline Joint & 0.5 & 1.7 & 1.7 & 13.1 & 0.6 & 0.7 \\
\hline Spontaneous joint & 0.0 & 1.0 & 1.0 & 9.2 & 0.0 & 0.0 \\
\hline Median dosing interval (d) & 3.5 & 7.0 & 5.0 & NR/NA & 3.5 & 3.5 \\
\hline Median CFC/wk (IU/kg) & 79.5 & 65.7 & 70.6 & NR/NA & 101.9 & 94.9 \\
\hline Median CFC/y (IU/kg) & 4223 & 3510 & NR/NA & $671^{\mathrm{e}}$ & 5418 & 4990 \\
\hline
\end{tabular}

$\overline{A B R}$ annualized bleeding rate, $C F C$ clotting factor consumption, $d$ days, $E T$ episodic treatment, $I P$ individualized prophylaxis, $I U$ international unit, $M P$ modified prophylaxis, pts patients, $w k$ week, WP weekly prophylaxis, $y$ year, NR/NA not reported or not available/applicable

${ }^{a}$ Median treatment duration: $4.5 \mathrm{y}$ in A-LONG/ASPIRE (3.9 y in ASPIRE only) and $3.5 \mathrm{y}$ in Kids A-LONG/ASPIRE (3.2 y in ASPIRE only)

${ }^{\mathrm{b}}$ Three pts received MP, including one in the $<6$ y age cohort initially assigned to IP who subsequently switched to MP (data not shown)

'21 pts who were initially assigned to IP, WP or ET subsequently switched to MP

${ }^{\mathrm{d}}$ In A-LONG, $n=72,16,16$ and 11 for IP, WP, MP and ET, respectively. In Kids A-LONG, $n=2$ and 5 for the $<6$ y and 6-11 y age cohorts

${ }^{\mathrm{e}}$ In six pts who received ET for $\geq 1$ year in ASPIRE 
The low ABRs and extended dosing intervals achieved in A-LONG and Kids A-LONG were maintained in the majority of patients who received efmoroctocog alfa IP in the 4-year ASPIRE extension study [13] (Table 3). Among adults and adolescents enrolled from A-LONG, ABRs were lower in those receiving IP compared with WP and MP; ABRs in all three prophylaxis groups were lower than those in the ET group (Table 3). Compared with A-LONG, the dosing interval lengthened for $21 \%$ of adults and adolescents over the course of ASPIRE (23\% lengthened their dosing interval to $>5$ days); it shortened for $8 \%$ of patients. Median weekly FVIII consumption at the end of ASPIRE was the same as that at the end of A-LONG (75 IU/kg vs $75 \mathrm{IU} / \mathrm{kg} ; n=128)$ [13]. Compared with Kids A-LONG, the dosing interval lengthened for $7 \%$ of children during ASPIRE [3\% (all aged $>6$ years) lengthened their dosing interval to $>5$ days]; it shortened for $5 \%$ of patients. In contrast to adults and adolescents, median weekly FVIII consumption in children was higher at the end of ASPIRE than at the end of Kids A-LONG (95 IU/ $\mathrm{kg}$ vs $75 \mathrm{IU} / \mathrm{kg} ; n=61$ ). Of note, $>99 \%$ of the 1680 physician global assessments of response to prophylactic treatment were graded 'excellent' (87\%) or 'effective' (13\%) [13].

In the final longitudinal analysis of patients treated prophylactically in the core and ASPIRE studies, the median cumulative treatment duration was 4.2 years in evaluable adults and adolescents from A-LONG and 3.4 years in evaluable children from Kids A-LONG (post hoc data) [19]. The median overall ABRs at years 1 and 5 were $1.2(n=122)$ and $0.5(n=46)$, respectively, in A-LONG/ASPIRE participants receiving efmoroctocog alfa IP, and $2.0(n=38)$ and $1.7(n=13)$ among those receiving efmoroctocog alfa WP. The median overall ABRs at years 1 and 4 were $1.8(n=61)$ and $1.3(n=31)$, respectively, in Kids A-LONG/ASPIRE participants who received efmoroctocog alfa IP [19].

Patients with pre-existing target joints treated prophylactically with efmoroctocog alfa in Kids A-LONG/ASPIRE and A-LONG/ASPIRE sustained low target joint and spontaneous target joint ABRs (in addition to low overall ABRs) and, in almost all cases, achieved target joint resolution (i.e. $\leq 2$ spontaneous bleeds in the joint in a 12-month period) [post hoc data] [20]. According to the final longitudinal analysis [20], the median spontaneous target joint ABR was zero in patients with pre-existing target joint(s) at entry into Kids A-LONG $(n=13)$ and A-LONG $(n=82)$ who received IP. Overall, $100 \%$ and $99.6 \%$ of target joints resolved in Kids A-LONG/ASPIRE ( $n=9$ evaluable $)$ and A-LONG/ ASPIRE ( $n=235)$, respectively; of those target joints with $\geq 6$ months of follow-up post-resolution, $100 \%$ and $95 \%$ showed no target joint reoccurrence, respectively [20].

Importantly, improvements in joint health have been observed over time in patients receiving efmoroctocog alfa prophylaxis in Kids A-LONG/ASPIRE and A-LONG/ ASPIRE, as assessed using the Haemophilia Joint Health
Score (HJHS) and modified HJHS (mHJHS), respectively (post hoc data) [20-22]. For example, significant improvements in HJHS ( $p<0.05$ vs Kids A-LONG baseline; $n=24$ evaluable patients) [22] and mHJHS ( $p=0.001$ vs A-LONG baseline; $n=47$ ) [21] were seen at ASPIRE year 2. There were improvements regardless of whether or not patients had target joints at Kids A-LONG/A-LONG baseline (present vs absent) and irrespective of the pre-study FVIII treatment regimen (prophylactic vs episodic) [21], although the data reported indicate that the benefits are greater in patients with pre-existing target joints and those on pre-study ET [21, 22]. In the final longitudinal analysis of Kids A-LONG/A-LONG and ASPIRE [20], the mean change in HJHS from Kids A-LONG baseline (1.5) to the end of ASPIRE was -1.0 ( $n$ $=42$ evaluable patients; median of 3.69 years of follow-up); the mean change in mHJHS from A-LONG baseline (21.6) to the end of ASPIRE was $-3.5(n=78 ; 3.72$ years $)$.

Regarding longer-term HRQOL, there were statistically significant $(p<0.05)$ and clinically meaningful improvements in HRQOL from Kids A-LONG baseline to ASPIRE year 2, as measured using the child- and parent-reported Canadian Hemophilia Outcomes-Kids Life Assessment Tool ( $n=16$ evaluable patients) [22].

\subsubsection{Treatment of Acute Bleeding Episodes}

Efmoroctocog alfa was effective in controlling acute bleeding in the A-LONG, Kids A-LONG and ASPIRE studies [6, $7,11-13,23]$. The majority of acute bleeds were controlled with one $(87 \%, 81 \%$ and $\geq 76 \%$ of episodes in A-LONG [7, 11, 23], Kids A-LONG [7, 12] and ASPIRE [13], respectively) or up to two $(98 \%[7,11,23], 93 \%$ [7, 12] and $\geq 93 \%$ [13], respectively) injections of efmoroctocog alfa; the median dose per injection to treat a bleeding episode was 27.4 [11], 49.7 [12] and $\geq 26.4$ [13] IU/kg, respectively. The majority of responses to first injections were rated by patients as 'excellent' or 'good' $(78 \%$ [7, 11, 23], 93\% [7] and $\geq 73 \%$ [13], respectively).

\subsubsection{Perioperative Management}

Efmoroctocog alfa effectively provided perioperative haemostatic control across a wide spectrum of major and minor surgeries in A-LONG, Kids A-LONG and ASPIRE [13, 24, 25] (post hoc data [25]). In total, 46 major and 90 minor surgeries were performed on 32 and 70 patients, based on the final pooled analysis of these three studies [25]. Patients undergoing surgery received an investigator-determined efmoroctocog alfa regimen; it was administered on the day of surgery for 44 major (including 33 orthopaedic and 2 spinal surgeries) and 84 minor procedures. During most major $(87 \%)$ and minor (89\%) surgeries, haemostasis was maintained with $\leq 1$ efmoroctocog alfa injection administered 
on the day of surgery or the day prior to surgery (i.e. loading dose). In addition, all major and minor surgeries evaluated for haemodynamic response were rated by investigators/surgeons as being 'excellent' [i.e. intraoperative and postoperative blood loss similar to, or less than, that for a non-haemophilic patient: $93 \%$ and $85 \%$ of major and minor surgeries, respectively] or good (i.e. blood loss increased, but not to a clinically significant extent, over expectations for a non-haemophilic patient). The median total dose of efmoroctocog alfa to maintain haemostasis was 61 and 62 $\mathrm{IU} / \mathrm{kg}$ during major and minor surgeries, respectively. Blood products were transfused for 4 major surgeries: bilateral knee arthroplasty, above-the-knee amputation, unilateral knee arthroplasty and unilateral hip arthroplasty [25].

\subsubsection{Real-World Experience}

The effectiveness of prophylactic treatment with efmoroctocog alfa in PTPs of all ages with haemophilia A in clinical practice is being evaluated in three ongoing, prospective, observational, phase IV studies being conducted at multiple centres across Germany (PREVENT [26, 27]), Europe (A-SURE [28, 29]; see Sect. 6), and Europe and the Middle East (A-MORE [30]; see Sect. 6).

The first interim analyses of patients observed for $\geq 9$ months in the 24-month, noncomparative PREVENT study showed that efmoroctocog alfa prophylaxis maintained good protection from bleeds with a low injection frequency [26, 27]. For all 67 evaluable patients (mean age 26.2 years; $96 \%$ with severe disease), the median overall ABR was 0.9; the median weekly efmoroctocog alfa injection frequency and dose were 2.2 and $84.6 \mathrm{IU} / \mathrm{kg}$ [26]. For the 55 evaluable paediatric patients aged $<18$ years (median age 8.0 years; $89 \%$ with severe disease), the median overall ABR was 0.6; the median weekly efmoroctocog alfa injection frequency and dose were 2.3 and $102 \mathrm{IU} / \mathrm{kg}$ [27].

A large number of retrospective real-world studies [31-52] have confirmed the efficacy of efmoroctocog alfa in the prophylaxis of bleeding observed in phase III clinical trials. The following discussion, however, only focuses on those involving $>30$ PTPs from one or more European countries [31, 36-44, 47-49] or the USA [50-52].

In before-after (within-patient) studies, transitioning to prophylaxis with efmoroctocog alfa from that with conventional FVIII products [including standard half-life (SHL) rFVIII preparations] was associated with maintained or lowered ABRs, while FVIII injection frequency and FVIII consumption were decreased (Table 4). Across the studies, changes in mean or median ABRs or bleed rates ranged from zero (i.e. no change) up to reductions of four ( $p \leq 0.003$ vs previous prophylaxis [40, 41, 47, 49]); these were accompanied by reductions in the median number of weekly or yearly injections ranging from 22 to $33 \%$ ( $p<0.001$ vs previous prophylaxis
$[40,41,44,48,50])$ and reductions in weekly, monthly or yearly FVIII consumption typically ranging from 10 to $40 \%$ ( $p$ $\leq 0.028$ vs previous prophylaxis $[31,40,44,49,50])$ (Table 4$)$.

Across comparative studies, ABRs with efmorocto$\operatorname{cog}$ alfa prophylaxis were comparable to, or lower than, those with SHL FVIII products, and higher proportions of efmoroctocog alfa recipients experienced zero bleeds (Table 5). FVIII injection frequency was also numerically reduced with efmoroctocog alfa relative to SHL FVIII products (Table 5). Bleeding outcomes and dosing data for efmoroctocog alfa were generally similar to those for the other EHL rFVIII products included in these studies (Table 5). In the MOTHIF-II study [48, 49], the mean ABR decreased significantly in patients with severe disease who switched from conventional FVIII prophylaxis to efmoroctocog alfa prophylaxis $(n=25$; Table 4$)$, but remained stable in those who, instead of switching, continued to receive conventional FVIII prophylaxis $(n=49)$. FVIII consumption decreased significantly in patients who switched to efmoroctocog alfa prophylaxis (Table 4 ), but increased by $\approx 10 \%$ in those remaining on conventional FVIII prophylaxis [49].

The effectiveness of efmoroctocog alfa for perioperative haemostasis in clinical practice in Europe has also been confirmed in retrospective real-world studies in a total of 47 PTPs with severe haemophilia A (35 from Ireland [53]; 12 from Nordic countries [54]) who underwent a total of 15 major and 80 minor surgeries [53, 54]. For major procedures, adequate haemostasis on the day of surgery was achieved with one or two efmoroctocog alfa injections; most minor procedures were managed with a single injection [53].

\subsection{Previously Untreated Patients}

The efficacy of efmoroctocog alfa in the prevention and treatment of bleeding episodes in previously untreated patients (PUPs) has been evaluated in an open-label, noncomparative, multinational, phase III trial that enrolled males aged $<6$ years and weighing $\geq 3.5 \mathrm{~kg}$ with severe haemophilia A (PUPs A-LONG) [55, 56].

A total of 103 participants $(77.7 \%$ aged $<1$ year; $19.4 \%$ with a family history of inhibitors; $79.6 \%$ with a high-risk haemophilia genotype) received $\geq 1$ dose of efmoroctocog alfa; these included 81 who started on ET [of whom 69 subsequently switched to prophylaxis (suggested initial regimen of $25-80 \mathrm{IU} / \mathrm{kg}$ every 3-5 days)] and 20 who started on prophylaxis. Two patients were not assigned a regimen. Patients were followed for up to 3 years. The primary endpoint was the development of inhibitors; secondary endpoints included bleed control (e.g. ABRs and resolution of acute bleeding episodes) [55].

Efmoroctocog alfa provided effective bleed control in this paediatric patient population [55]. With prophylactic treatment (for a median of 44 weeks), ABRs were low (1.5 overall; zero 
Table 4 Real-world effectiveness of switching to prophylaxis with efmoroctocog alfa from conventional FVIII products in patients with haemophilia A: results of before-after (within-patient) studies from Europe and the USA

\begin{tabular}{|c|c|c|c|c|}
\hline \multirow[t]{2}{*}{ Study (no. of pts) } & \multirow[t]{2}{*}{ Country } & \multicolumn{3}{|c|}{ Outcome post-switch [pre-switch value] } \\
\hline & & $\mathrm{ABR}^{\mathrm{a}}$ & $\mathrm{IF}^{\mathrm{a}}$ & $\mathrm{CFC}^{\mathrm{a}}$ \\
\hline Benitez et al. [31] (40) & Spain & $\begin{array}{l}\downarrow 51 \% * * \mathrm{~b} \\
\downarrow 55 \% * * \mathrm{~b}, \mathrm{c}\end{array}$ & & $\mathrm{IU} / \mathrm{pt} / \mathrm{mo}: \downarrow 40 \% * \mathrm{~b}, \mathrm{c}$ \\
\hline Carcao et al. [50] (38) & USA & $1\left(0^{\mathrm{d}}\right)\left[1.2\left(0^{\mathrm{d}}\right)\right]$ & /wk: $2.3 * * *[3.5]$ & IU/kg/wk: 85.5** [103] \\
\hline Giraud et al. [41] (34) & France & $\begin{array}{l}\text { All pts: } 1 * * *[5] \\
\text { pts < } 12 \text { y: } 3 *[7.5] \\
\text { pts } 12-18 \text { y: } 2 *[6] \\
\text { pts }>18 \text { y: } 0 * *[1]\end{array}$ & $\begin{array}{l}\text { /y: } 105 * * *[139] \\
/ \mathrm{y}: \downarrow 21 \% * \mathrm{~b} \\
/ \mathrm{y}: \downarrow 28 \% *{ }^{*} \mathrm{~b} \\
/ \mathrm{y}: \downarrow 18 \% * * \mathrm{~b}\end{array}$ & $\begin{array}{l}\mathrm{IU} / \mathrm{pt} / \mathrm{y}: \downarrow^{\mathrm{b}} \\
\mathrm{IU} / \mathrm{pt} / \mathrm{y}: \uparrow 6 \%^{\mathrm{b}} \\
\mathrm{IU} / \mathrm{pt} / \mathrm{y}: \downarrow^{\mathrm{b}} \\
\mathrm{IU} / \mathrm{pt} / \mathrm{y}: \uparrow 2 \%^{\mathrm{b}}\end{array}$ \\
\hline Holmström et al. [37] (83e) & Sweden & $0[0]$ & /wk: $2.8[3.8]$ & IU/kg/wk: $79.0[87.9]$ \\
\hline Horvais et al. $[48,49]\left(85^{f}\right)$ & France & $4 * *[6.6]$ & /wk: $2 * * *[3]$ & $\mathrm{IU} / \mathrm{pt} / \mathrm{y}: \downarrow 40 * \%^{\mathrm{b}}$ \\
\hline Katsarou et al. [47] (42) & Greece & $1.5 * *[3.1]$ & & \\
\hline Morais et al. [39] (35) & Portugal & $0^{\mathrm{g}}\left(0^{\mathrm{d}, \mathrm{g}}\right)\left[2^{\mathrm{g}}\left(1^{\mathrm{d}, \mathrm{g}}\right)\right]$ & & $\mathrm{IU} / \mathrm{pt}: \downarrow 18.7 \%{ }^{\mathrm{b}, \mathrm{f}}$ \\
\hline Myren et al. [43] (87) & France, Germany, Italy, UK & & /wk: $2.1[3]$ & IU/pt/y: $\downarrow^{27 \%^{b}}$ \\
\hline Simon et al. [44] (34) & Italy & $0.13[0.63]$ & $/ \mathrm{y}: 122 * * *[156]$ & $\mathrm{IU} / \mathrm{pt} / \mathrm{y}: \downarrow 28 \% * * * \mathrm{~b}$ \\
\hline van der Sluijs et al. [42] (113) & France, Germany, Italy, Spain, UK & $1[3]$ & /wk: $2[3]$ & IU/kg/wk: 86 [103.3] \\
\hline Wall et al. [40] (158) & UK & $2 * *\left(1^{* * d}\right)\left[2.6\left(1^{\mathrm{d}}\right)\right]$ & /wk: $2.3 * *[3.2]$ & IU/kg/wk: $68^{* *}[81]$ \\
\hline
\end{tabular}

(A)BR (annualized) BR, (A)JBR (annualized) joint BR, $B R$ bleeding rate, $C F C$ clotting factor consumption, $I F$ injection frequency, $I U$ international unit, mo month, $p t(s)$ patient(s), wk week, $y$ year, $\uparrow$ increased, $\downarrow$ decreased

$* p \leq 0.043, * * p \leq 0.004, * * * p \leq 0.0007$ vs pre-switch

${ }^{a}$ Mean (ABR [31, 44, 47-49], IF [37, 43], CFC [37, 39, 42, 43]) or median (ABR [37, 40-42, 50], BR [39], JBR [39], AJBR [40, 50], IF [40-42, 44, 50], CFC $[40,41,44,50])$ value, where known

${ }^{\mathrm{b}} \mathrm{vs}$ pre-switch value

${ }^{\mathrm{c}}$ Analysis includes an additional $11 \mathrm{pts}$ who received on-demand efmoroctocog alfa

${ }^{\mathrm{d} J B R}[39]$ or AJBR [40, 50]

$\mathrm{e}_{n}=62$ for $\mathrm{ABR}$

${ }^{\mathrm{f}} n=25$ for $\mathrm{ABR}$ and $\mathrm{CFC}$

${ }^{\mathrm{g}}$ Over a mean period of 10.6 mo [39]

for spontaneous and spontaneous joint bleeding episodes), the median dosing interval was 3.9 days, and the median yearly FVIII consumption was $5384 \mathrm{IU} / \mathrm{kg}$. With ET (administered for a median of 23.5 weeks), ABRs were 2.24 overall and zero for spontaneous and spontaneous joint bleeding episodes; the median yearly FVIII consumption was 198 IU/kg. A median of one efmoroctocog alfa injection was required for resolution of a bleeding episode; the majority ( $\geq 80 \%$ ) of responses to treatment were rated by patients as being 'excellent' or 'good' $[55,56]$.

\section{Tolerability of Efmoroctocog Alfa}

Efmoroctocog alfa was generally well tolerated and showed a consistent overall safety profile in the phase III A-LONG, Kids A-LONG [12] and ASPIRE [13] studies in PTPs with severe haemophilia A [11-13]. Across these studies, 276 adults, adolescents and children $[182(66 \%)$ aged $\geq 18$ years; $25(9 \%)$ aged $12-17$ years; and $69(25 \%)$ aged $<12$ years] received $\geq 1$ dose of efmoroctocog alfa as part of either routine prophylaxis, episodic (on-demand) treatment of bleeding episodes or perioperative management. These patients, of whom 200, 151 and 107 were treated for $\geq 104,156$ and 208 weeks, respectively, received a total of 82,024 injections, with a median of 303.5 (range 1-755) injections per patient. The total number of EDs was 80,848 , with a median of 294 (range 1-735) EDs per patient. Adverse reactions (ARs), including the development of inhibitors (the primary endpoint in Kids A-LONG [12] and ASPIRE [13]), were monitored for a total of 894 patient-years [6, 7].

Eleven (4\%) of the 276 patients reported ARs, the most frequent being arthralgia, headache, myalgia, malaise and rash [each reported by two $(0.7 \%)$ patients]. Other ARs [each reported in one $(0.4 \%)$ patient] included angiopathy (vascular pain after injection of efmoroctocog alfa), back pain, bradycardia, chest pain, cough, dizziness, dysgeusia, feeling cold, feeling hot, hot flush, hypertension, joint swelling, lower abdominal pain, and procedural hypotension. No age-specific differences in ARs were seen between adult and pediatric patients. Two patients withdrew from study due to ARs (arthralgia and rash) [6, 7].

No patients developed a neutralizing antibody to FVIII over the course of the A-LONG, Kids A-LONG or ASPIRE studies [6, 11-13]. A 25-year-old patient tested positive for a low-titre $(0.73 \mathrm{BU} / \mathrm{mL})$ inhibitor at week 14 in A-LONG, 
Table 5 Real-world effectiveness of prophylaxis with efmoroctocog alfa versus other recombinant FVIII products in patients with haemophilia A: results of multicentre, retrospective, chart-review studies from Europe and the USA

\begin{tabular}{|c|c|c|c|c|c|}
\hline Study (Country) & Comparators (no. of pts) & $\mathrm{ABR}^{\mathrm{a}}$ & 0 bleeds (\% pts) & $\mathrm{IF} / \mathrm{wk}(\leq 2 \mathrm{vs} \geq 3 ; \% \mathrm{pts})$ & $\mathrm{CFC}(\mathrm{IU} / \mathrm{kg} / \mathrm{wk})^{\mathrm{a}}$ \\
\hline \multirow{4}{*}{$\begin{array}{l}\text { Mancuso et al. [38] } \\
\text { (Italy) }\end{array}$} & Efmoroctocog alfa (73) & 0.0 & NR & 76.7 vs 23.3 & 92.3 \\
\hline & Lonoctocog alfa (60) & 0.0 & NR & 56.7 vs 43.3 & 91.6 \\
\hline & Octocog alfa $\left(\right.$ Advate $\left.^{\circledR}\right)(83)$ & 1.0 & NR & 16.9 vs 83.1 & 113.2 \\
\hline & Octocog alfa $\left(\right.$ Kovaltry $\left.^{\circledR}\right)(74)$ & 0.0 & NR & 36.5 vs 63.5 & 100 \\
\hline \multirow{5}{*}{$\begin{array}{l}\text { Olivieri et al. [36] } \\
\text { (Germany) }\end{array}$} & Efmoroctocog alfa (47) & 0.0 & 78.7 & 76.5 vs 23.5 & 87.0 \\
\hline & Lonoctocog alfa (40) & 0.0 & 75 & 82.5 vs 17.5 & 61.5 \\
\hline & Octocog alfa (Advate $\left.{ }^{\circledR}\right)(58)$ & 0.0 & 58.6 & 37.9 vs 62.1 & 87.5 \\
\hline & Octocog alfa (Kovaltry $\left.{ }^{\circledR}\right)(40)$ & 0.0 & 55 & 35 vs 65 & 96.3 \\
\hline & Moroctocog alfa (40) & 0.0 & 57.5 & 50 vs 50 & 79 \\
\hline \multirow{2}{*}{$\begin{array}{l}\text { Shrestha et al. [52] } \\
\text { (USA) }\end{array}$} & Efmoroctocog alfa (83) & $1.8[3.3$ at $\mathrm{BL}]$ & NR & NR & NR \\
\hline & Lonoctocog alfa (118) & $2.8[3.5$ at $\mathrm{BL}]$ & NR & NR & NR \\
\hline \multirow{6}{*}{$\begin{array}{l}\text { Yan et al. [51] } \\
\text { (USA) }\end{array}$} & Efmoroctocog alfa (40) & 2.0 & 22.5 & 65 vs 35 & 108.5 \\
\hline & Lonoctocog alfa (40) & 2.0 & 25 & 70 vs 30 & 91.9 \\
\hline & Rurioctocog alfa pegol (40) & 3.0 & 17.5 & 72.5 vs 27.5 & 97.6 \\
\hline & Octocog alfa $\left(\right.$ Advate $\left.^{\circledR}\right)(40)$ & 2.0 & 17.5 & 25 vs 75 & 114.0 \\
\hline & Octocog alfa (Kovaltry $\left.{ }^{\circledR}\right)(40)$ & 3.0 & 10 & 47.5 vs 52.5 & 95.1 \\
\hline & rFVIII-FS (Kogenate $\left.{ }^{\circledR}\right)(40)$ & 2.0 & 7.5 & 40 vs 60 & 102.5 \\
\hline
\end{tabular}

Pts had been treated for $\geq 8$ wks [36, 38, 51] or $\geq 6$ months [52] at the time of data collection

$A B R$ annualized bleeding rate, $B L$ baseline, $C F C$ clotting factor consumption, $I F$ injection frequency, $I U$ international unit, $N R$ not reported, pts patients, $r F V I I I$ recombinant FVIII, $r F V I I I-F S$ sucrose-formulated rFVIII, $w k(s)$ week(s)

$* p=0.001$ vs rFVIII

${ }^{a}$ Median values, except for Shrestha et al. [52] (average values)

but had negative results upon repeat testing 18 days later and thereafter [7]. No events of anaphylaxis were reported [6].

Efmoroctocog alfa was also generally well tolerated in the phase III PUPs A-LONG study in paediatric PUPs with severe haemophila A [55] (Sect. 3.2). The primary endpoint was the rate of total inhibitor development (calculated by dividing the number of patients who developed an inhibitor by the combined number of patients with $\geq 10$ EDs plus those with $<10$ EDs who had developed an inhibitor). Inhibitors developed in $28(31.1 \%)$ of 90 patients (87 with $\geq 10$ EDs and 3 with $<10$ EDs and an inhibitor); high-titre $(\geq 5 \mathrm{BU} / \mathrm{mL})$ inhibitors developed in $14(15.6 \%)$ of the 90 patients. The median time to inhibitor development was 9 (range 1-53) EDs. Other serious adverse events included deep vein/central venous access devices-associated thromboses $(n=2)$ and soft tissue haemorrhage occurring in the context of high-titre inhibitor development $(n=1)$ [55].

Results for inhibitor development in phase III clinical trials are supported by the findings of real-world studies [57, 58]. The FACTs study [57], for example, is evaluating the effectiveness of efmoroctocog alfa in adolescents and children aged $<18$ years with haemophilia A in Japan. In part 1 of this 2-year, prospective, multicentre, observational study, two of the 16 PUPS who were included in the second interim analysis developed low-titre inhibitors [57]. In a retrospective US study, one of two PUPs with haemophilia A developed a low-titre inhibitor $(1.4 \mathrm{BU} / \mathrm{mL})$ after 10 exposures to efmoroctocog alfa, although this resolved (i.e. anti-FVIII $<0.6 \mathrm{BU} /$ $\mathrm{mL}$ ) within eight additional exposures [58]. As expected, none of 41 PTPs with haemophilia A developed an inhibitor after initiating efmoroctocog alfa (range 24-432 EDs) [58].

\section{Dosage and Administration}

Efmoroctocog alfa is approved for the treatment and prophylaxis of bleeding in patients with haemophilia $\mathrm{A}$ in various countries worldwide, including those of the EU [6], as well as the USA [7] and Japan [8] (Sect. 1). Individual patients may vary in their pharmacokinetic and clinical responses to FVIII replacement therapy with efmoroctocog alfa; the dose and duration of substitution therapy depends on the severity of the FVIII deficiency, on the location and extent of bleeding and the patient's clinical condition [6-8]. Likewise, regimens for long-term prophylaxis vary by region and by patient age group [6-8].

Local prescribing information should be consulted for guidelines for efmoroctocog alfa regimens for the treatment of bleeding episodes, perioperative management and longterm prophylaxis, as well as for further information, including special warnings and precautions and contraindications. 


\section{Place of Efmoroctocog Alfa in the Management of Haemophilia A}

The efficacy and safety of efmoroctocog alfa for the longterm prophylaxis, acute treatment and perioperative management of bleeding in male PTPs of all ages with severe haemophilia A was initially demonstrated in phase III clinical trials, including two parent studies (A-LONG and Kids A-LONG) and their extension study (ASPIRE) (Sects. 3.1, 4). Due to flexibility in efmoroctocog alfa dosing, the design of the 4-year ASPIRE study approximated real-world practice [13]. The effectiveness of prophylaxis with this EHL rFVIII preparation in PTPs in clinical practice has subsequently been substantiated in an interim analysis of a prospective real-world study (PREVENT), as well as a large number of retrospective real-world studies (Sect. 3.1.4). Final findings from PREVENT are awaited with interest, as are the results of other ongoing, prospective, real-world studies (A-SURE; A-MORE) (Sect. 3.1.4). As anticipated-and consistent with the results of the phase III studies-no PTPs who received efmoroctocog alfa in a real-world study developed inhibitors (Sect. 4). Of note, the original efmoroctocog alfa population pharmacokinetic model used to design IP dosing regimens was based on clinical trial data in PTPs aged $\geq 12$ years with severe haemophilia A (including A-LONG participants) [59]. This model has since been expanded to include PTPs aged $<12$ years with severe haemophilia A (based on data from Kids A-LONG) [60]. It has also been externally validated in an expanded, alternative model constructed from real-world data in patients aged $\geq 5$ years [61].

Available data from A-LONG, Kids A-LONG and ASPIRE have confirmed the clinical benefits of FVIII prophylaxis (with efmoroctocog alfa) beyond low bleeding rates in terms of improving joint health and HRQOL in PTPs with severe haemophilia A (Sect. 3.1.1). The longterm effectiveness of prophylaxis with efmoroctocog alfa on joint health is being assessed further in a real-world setting in A-MORE, a 48-month, prospective, noncomparative study in $\approx 300$ PTPs of all ages with haemophilia A of any severity. Primary endpoints include target joint development, resolution and recurrence; secondary endpoints include Hemophilia Early Arthropathy Detection with Ultrasound (HEAD-US) score and HJHS (Sect. 3.1.4) [30]. As regards the effects of prophylaxis with efmoroctocog alfa on longterm HRQOL outcomes, fully published data from adults and adolescents treated in A-LONG/ASPIRE are awaited with interest, as are final findings from children treated in Kids A-LONG/ASPIRE. Real-world studies assessing the impact of efmoroctocog alfa prophylaxis on HRQOL are also desirable.

The efficacy and safety of efmoroctocog alfa in the prevention and treatment of bleeding episodes in male PUPs aged $<6$ years with severe haemophilia $\mathrm{A}$ has also been demonstrated in a phase III clinical trial (PUPs A-LONG) [Sect. 3.2]. Of note, the rate of total inhibitor development in this study $(\approx 30 \%$; Sect. 4$)$ was within the expected range, while that of high-titre inhibitors $(\approx 16 \%$; Sect. 4$)$ was reportedly lower than that previously reported in the literature [55]. Immune tolerance induction (ITI) is the only proven strategy to eradicate high-titre inhibitors, although it is both burdensome and costly, often requiring long-term, frequent administration of high(er) doses of FVIII [62]. As such, decreasing ITI treatment time may diminish the negative impact of inhibitor development on patients' health and HRQOL, as well as reduce the FVIII consumption and related cost associated with this approach [63]. While the immunogenicity of efmoroctocog alfa does not appear to be dissimilar to that of other FVIII products [58], there is evidence from preclinical and retrospective real-world studies to suggest that this EHL rFVIII preparation may allow relatively rapid ITI, possibly due to immunomodulatory effects (upregulation of regulatory $\mathrm{T}$ cells and promotion of FVIII-specific T-cell tolerance) attributed to the Fc domain of the molecule $[9,64,65]$.

In support of the retrospective data, interim results from a prospective, open-label, multicentre study evaluating efmoroctocog alfa for initial ITI in patients of any age with severe haemophilia A and high-titre inhibitors (verITI-8) indicate that this EHL rFVIII preparation may indeed offer a rapid time to tolerization in some individuals [63]. At the time of this analysis, 6 of 14 patients receiving $\geq 1$ dose of efmoroctocog alfa for ITI have been successfully tolerized, including five in $\leq 12.5$ weeks. Seven patients are continuing ITI [median time on ITI of 16.0 (range 0.1-35.6) weeks], and one has failed [63]. Final findings from this ongoing study are awaited with interest. Furthermore, the final results of another prospective, open-label, multicentre study (ReITIrate) indicated a potential clinical benefit of efmoroctocog alfa for rescue ITI in patients of any age with severe haemophilia A and high-titre inhibitors who had failed previous ITI attempts [66]. Of the 16 patients enrolled, nine completed the ITI period (i.e. treatment with efmoroctocog alfa 200 $\mathrm{IU} / \mathrm{kg} /$ day for $\leq 60$ weeks), including four who reached a confirmed negative inhibitor titre $(<0.6 \mathrm{BU} / \mathrm{mL})$ within a median of 19 (range 11-60) weeks. One of the four patients reaching a confirmed negative inhibitor titre was a complete ITI success [66].

Current guidelines do not recommend any one EHL or SHL FVIII preparation over another [3, 67]. In the absence of direct randomized, head-to-head trials, indirect comparisons based on published clinical trials (typically pivotal studies) suggest that prophylaxis with efmoroctocog alfa requires fewer injections and less weekly FVIII consumption to achieve similar or improved ABRs relative to conventional rFVIII products (octocog alfa, moroctocog alfa, 
simoctocog alfa and turoctocog alfa) [68], and has comparable [69] (or improved [70]) efficacy and FVIII consumption relative to other EHL rFVIII preparations (i.e. lonoctocog alfa [69], damoctocog alfa pegol [69, 70], rurioctocog alfa pegol [69] and turoctocog alfa pegol [69]).

These results are supported by retrospective real-world studies in which prophylaxis with efmoroctocog alfa achieved comparable or lower ABRs with fewer injections and, in several cases, less FVIII consumption, relative to conventional rFVIII preparations, and showed comparable efficacy and, in some instances, broadly similar FVIII consumption, to other EHL rFVIII preparations (Sect. 3.1.4). Currently, the 24-month, prospective A-SURE study is comparing the effectiveness and real-world usage of efmoroctocog alfa versus conventional FVIII products for prophylaxis in PTPs of all ages with haemophilia A. Primary endpoints include ABR, injection frequency and FVIII consumption $[28,29]$. Positive results from this and other prospective studies designed to show that the potential advantages of EHL over SHL FVIII products (e.g. improved adherence, enhanced HRQOL and increased bleed protection; Sect. 1) are actually realized in a real-world setting, will help define the relative role of EHL FVIII products such as efmorocto$\operatorname{cog}$ alfa in the treatment of haemophilia A.

In practice, FVIII product choice may be influenced not only by pharmacokinetic differences and patient preference, but also by cost [67]. Semi-Markov models incorporating joint health data indicate that, for the life-time treatment of severe haemophilia A patients without inhibitors, prophylaxis with efmoroctocog alfa is cost effective relative to SHL rFVIII from an Italian National Health Service perspective [71] and relative to both SHL rFVIII and rurioctocog alfa pegol from a US healthcare payer perspective [72].

In conclusion, efmoroctocog alfa is an established and effective EHL FVIII replacement therapy for the management of haemophilia A.

\section{Data Selection Efmoroctocog alfa: 526 records identified}

Search strategy: EMBASE, MEDLINE and PubMed from 2016 to present. Previous Adis Drug Evaluation published in 2016 was hand-searched for relevant data. Clinical trial registries/databases and websites were also searched. Key words were efmoroctocog alfa, ELOCTATE, ELOCTA, recombinant Factor VIII Fc, haemophilia A. Records were limited to those in English language. Searches last updated 21 Oct 2021

Supplementary Information The online version contains supplementary material available at https://doi.org/10.1007/s40265-021-01615-w.

Acknowledgments During the peer review process, the manufacturer of efmoroctocog alfa was also offered an opportunity to review this article. Changes resulting from comments received were made on the basis of scientific and editorial merit.

\section{Declarations}

Funding The preparation of this review was not supported by any external funding.

Authorship and Conflict of interest James E. Frampton is a salaried employee of Adis International Ltd/Springer Nature, and declares no relevant conflicts of interest. All authors contributed to the review and are responsible for the article content.

Ethics approval, Consent to participate, Consent to publish, Availability of data and material, Code availability Not applicable.

Open Access This article is licensed under a Creative Commons Attribution-NonCommercial 4.0 International License, which permits any non-commercial use, sharing, adaptation, distribution and reproduction in any medium or format, as long as you give appropriate credit to the original author(s) and the source, provide a link to the Creative Commons licence, and indicate if changes were made. The images or other third party material in this article are included in the article's Creative Commons licence, unless indicated otherwise in a credit line to the material. If material is not included in the article's Creative Commons licence and your intended use is not permitted by statutory regulation or exceeds the permitted use, you will need to obtain permission directly from the copyright holder. To view a copy of this licence, visit http://creativecommons.org/licenses/by-nc/4.0/.

\section{References}

1. Ar MC, Balkan C, Kavaklı K. Extended half-life coagulation factors: a new era in the management of hemophilia patients. Turk $\mathbf{J}$ Haematol. 2019;36(3):141-54.

2. Frampton JE. Efmoroctocog alfa: a review in haemophilia A. Drugs. 2016;76(13):1281-91.

3. Srivastava A, Santagostino E, Dougall A, et al. WFH guidelines for the management of hemophilia, 3rd edition. Haemophilia. 2020;26(Suppl 6):1-158.

4. Lambert T, Benson G, Dolan G, et al. Practical aspects of extended half-life products for the treatment of haemophilia. Ther Adv Hematol. 2018;9(9):295-308.

5. Schiavoni M, Napolitano M, Giuffrida G, et al. Status of recombinant factor VIII concentrate treatment for hemophilia A in Italy: characteristics and clinical benefits. Front Med. 2019;6:261.

6. Swedish Orphan Biovitrum AB. Elocta: EU summary of product characteristics. 2021. https://www.ema.europa.eu. Accessed 10 Sep 2021.

7. Sanofi. Eloctate: US prescribing information. 2020. https://www. eloctate.com. Accessed 10 Sep 2021.

8. Sanofi. Eloctate ${ }^{\circledR}$ : Japanese prescribing information. 2019. https:// www.pmda.go.jp. Accessed 10 Sep 2021.

9. Meeks SL, Lacroix-Desmazes S. Emerging benefits of Fc fusion technology in the context of recombinant factor VIII replacement therapy. Haemophilia. 2020;03:03.

10. Pouplard C, Sattler L, Ryman A, et al. Multicentre pharmacokinetic evaluation of rFVIII-Fc (efmoroctocog alfa) in a real life and comparison with non-extended half-life FVIII concentrates. Haemophilia. 2020;26(2):282-9.

11. Mahlangu J, Powell JS, Ragni MV, et al. Phase 3 study of recombinant factor VIII Fc fusion protein in severe hemophilia A. Blood. 2014;123(3):317-25. 
12. Young G, Mahlangu J, Kulkarni R, et al. Recombinant factor VIII Fc fusion protein for the prevention and treatment of bleeding in children with severe hemophilia A. J Thromb Haemost. 2015;13(6):967-77.

13. Nolan B, Mahlangu J, Pabinger I, et al. Recombinant factor VIII Fc fusion protein for the treatment of severe haemophilia A: final results from the ASPIRE extension study. Haemophilia. 2020;26:494-502.

14. European Medicines Agency. Elocta assessment report. 2015. http://www.ema.europa.eu. Accessed 10 Sep 2021.

15. Shapiro A, Ragni M, Kulkarni R, et al. Recombinant factor VIII Fc fusion protein: extended-interval dosing maintains low bleeding rates and correlates with von Willebrand factor levels. J Thromb Haemost. 2014;12(11):1788-800.

16. Wyrwich K, Krishnan S, Auguste P, et al. Changes in healthrelated quality of life with treatment of longer-acting clotting factors: results in the A-LONG and B-LONG clinical studies. Haemophilia. 2016;22(6):866-72.

17. Pasi J, Hermans C, Hakimi Z, et al. Improvement in pain-related quality of life in patients with haemophilia A treated with RFVIIIFC individualized prophylaxis: post-hoc analysis from A-LONG [abstract no. ABS128]. Haemophilia. 2021;27(Suppl 2):ABS128.

18. Quon DV, Klamroth R, Kulkarni R, et al. Low bleeding rates with increase or maintenance of physical activity in patients treated with recombinant factor VIII Fc fusion protein (rFVII$\mathrm{IFc}$ ) in the A-LONG and Kids A-LONG Studies. Haemophilia. 2017;23(1):e39-42.

19. Konkle B, Young G, Liesner R, et al. Long-term efficacy and safety of prophylactic treatment with recombinant factor VIII Fc fusion protein (rFVIIIFc) in subjects with severe haemophilia A: final longitudinal analysis of A-LONG/kids A-LONG and ASPIRE [abstract no. P039]. Haemophilia. 2019;25(Suppl 1):P039.

20. Oldenburg J, Pasi J, Pabinger I, et al. Improvements in joint health during long-term use of recombinant factor VIII Fc fusion protein prophylaxis in subjects with haemophilia A [abstract no. P158]. Haemophilia. 2019;25(Suppl 1):P158.

21. Oldenburg J, Kulkarni R, Srivastava A, et al. Improved joint health in subjects with severe haemophilia A treated prophylactically with recombinant factor VIII Fc fusion protein. Haemophilia. 2018;24(No 1):77-84.

22. Blanchette V, Kulkarni R, Tsao E, et al. Longitudinal hemophilia joint health scores and life assessment (CHO-KLAT) in children with severe hemophilia A and long-term rFVIIIFc prophylaxis [abstract no. P290]. Blood. 2018; Abstract Suppl:467.

23. Shapiro AD, Mahlangu JN, Perry D, et al. Treatment of bleeding episodes with recombinant factor VIII Fc fusion protein in A-LONG study subjects with severe haemophilia A. Haemophilia. 2017;23(3):392-9.

24. Mahlangu JN, Ragni M, Gupta N, et al. Long-acting recombinant

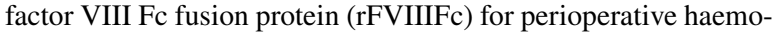
static management in severe haemophilia A. Thromb Haemost. 2016;116(07):1-8.

25. Chowdary P, Ragni MV, Feng, J, et al. Recombinant factor VIII $\mathrm{Fc}$ fusion protein (rFVIIIFc) efficacy for perioperative haemostatic management in severe haemophilia [abstract plus poster P-14]. In: WFH 16th international musculoskeletal congress. 2019.

26. Bidlingmaier C, Heller C, Schilling K, et al. First interim analysis of a 24-month, prospective, non-interventional, multicentre study in Germany evaluating the real-world usage and effectiveness of rFVIIIFc and rFIXFc in patients with haemophilia A or B (PREVENT) [abstract no. PB0952 plus poster]. Res Pract Thromb Haemost. 2020;4(Suppl 1):PB0952.

27. Heller $\mathrm{C}$, Bidlingmaier $\mathrm{C}$, Escuriola $\mathrm{C}$, et al. Interim analysis of the PREVENT study: real world prospective data from children and adolescents with haemophilia A or B treated with recombinant factor VIII Fc (rFVIIIFc) or recombinant factor IX $\mathrm{Fc}(\mathrm{rFIXFc})$ [abstract no. PB0506 plus poster]. Res Pract Thromb Haemost. 2020;4(Suppl 1):PB0506.

28. Oldenburg J, Hay CRM, Jimenez-Yuste V, et al. Design of a prospective observational study on the effectiveness and real-world usage of recombinant factor VIII Fc ( $\mathrm{rFVIIIFc)} \mathrm{compared} \mathrm{with}$ conventional products in haemophilia A: the A-SURE study. BMJ Open. 2019;9(5):e028012.

29. Hay C, Jimenez-Yuste V, Oldenburg J, et al. Baseline data from A-sure: a 24-month prospective, non-interventional study comparing effectiveness of recombinant factor VIII FC (rFVIIIFc) with conventional factor VIII (CONVFVIII) prophylaxis in haemophilia A [abstract no. P079]. Haemophilia. 2020;26(Suppl 2):P079.

30. Lethagen S, O'Malley M, Szamosi J. Rationale and study design for a prospective 48-month, multi-centre, observational study evaluating long-term effectiveness of RFVIIIFc on joint health-the A-MORE study [abstract no. ABS169]. Haemophilia. 2021;27(Suppl 2):ABS169.

31. Benitez O, Suito M, Cabirta A, et al. The impact of switching previously treated patients (PTPs) to recombinant factor VIII Fc fusion protein ( $\mathrm{rFVIIIFc)}$ in reducing the annualized bleeding rate (ABR): a single center experience [abstract no. PB0971]. Res Pract Thromb Haemost. 2020;4(Suppl 1):PB0971.

32. Brennan Y, Parikh S, McRae S, et al. The Australian experience with switching to extended half-life factor VIII and IX concentrates: on behalf of the Australian Haemophilia Centre Directors' Organisation. Haemophilia. 2020;26(3):529-35.

33. Tagliaferri A, Matichecchia A, Rivolta GF, et al. Optimising prophylaxis outcomes and costs in haemophilia patients switching to recombinant FVIII-Fc: a single-centre real-world experience. Blood Transfus. 2020;18(5):374-85.

34. Wang C, Young G. Clinical use of recombinant factor VIII Fc and recombinant factor IX $\mathrm{Fc}$ in patients with haemophilia $\mathrm{A}$ and $\mathrm{B}$. Haemophilia. 2018;24(3):414-9.

35. Peyvandi F, Garagiola I, Boscarino M, et al. Real-life experience in switching to new extended half-life products at European haemophilia centres. Haemophilia. 2019;25(6):946-52.

36. Olivieri M, Sommerer P, Maro G, et al. Assessing prophylactic use and clinical outcomes in hemophilia A patients treated with rVIIISingleChain and other common rFVIII products in Germany. Eur J Haematol. 2020;104(4):310-7.

37. Holmström M, Olsson E, Astermarket J, et al. Real-world prophylactic usage of recombinant factor VIII Fc in Sweden: a report from the Swedish national registry for bleeding disorders. Haemophilia. 2021;27(4):e554-8.

38. Mancuso ME, Santoro C, Maro G, et al. Comparison of FVIII prophylaxis treatment regimen and associated clinical outcomes between rVIII-singlechain and other rFVIII products commonly used in Italian hemophilia A patients [abstract no. PB0898]. Res Pract Thromb Haemost. 2020;4(Suppl 1):PB0898.

39. Morais $\mathrm{S}$, Campaniço $\mathrm{S}$, Coutinho M, et al. Optimizing outcomes in hemophilia A prophylaxis using recombinant factor VIII Fc fusion protein ( $\mathrm{rFVIIIFc)}$ ): results from three Portuguese haemophilia centers [abstract no. PB1158]. Res Pract Thromb Haemost. 2020;4(Suppl 1):PB1158.

40. Wall $\mathrm{C}$, Scott $\mathrm{M}$, Xiang $\mathrm{H}$, et al. Longitudinal analysis of $\mathrm{rFVIIIFC}$ use and efficacy in the UK: a report from the National Haemophilia Database [abstract no. PB0878]. Res Pract Thromb Haemost. 2020;4(Suppl 1):PB0878.

41. Giraud R, Delmotte N, Gensollen S, et al. Recombinant factor VIII Fc fusion protein ( $\mathrm{rFVIIIFc)}$ in real life: one-year clinical and economic outcomes. Drugs Real World Outcomes. 2021. https:// doi.org/10.1007/s40801-021-00259-2.

42. Van Der Sluijs M, Tawil S, Castelnuovo E, et al. A survey of physicians' treatment switching practice in long-term prophylaxis for 
people with haemophilia A in five European countries [abstract no. PB0692]. Res Pract Thromb Haemost. 2019;3(Suppl 1):PB0692.

43. Myren KJ, Van Der Sluijs M, Kritikou P, et al. Real world switching from conventional FVIII products to RFVIIIFC in France, Germany, Italy and the UK [abstract no.PF297 plus poster]. HemaSphere. 2018;2(Suppl 2):101.

44. Simon B, Gualtierotti R, Biguzzi E, et al. Switching to extended half-life recombinant factor VIII (rFVIII-Fc) in patients with hemophilia A: data from a single-center cohort [abstract]. Blood Transfus. 2020;18(Suppl 4):S503-4.

45. Goldmann G, Marquardt N, Horneff S, et al. Real-world clinical experience of extended half-life recombinant factor VIII Fc fusion protein $(\mathrm{rFVIIIFc})$ in comparison to conventional factor products in patients with severe hemophilia A. CMRO. 2021;4(5):950-60.

46. Ay C, Feistritzer C, Rettl J, et al. Bleeding outcomes and factor utilization after switching to an extended half-life product for prophylaxis in haemophilia A in Austria. Sci Rep. 2021;11:12967.

47. Katsarou O, Kouramba A, Nomikou E, et al. Real world data on haemophilia A patients switching to rFVIIIFc treatment in Greece. Interim results from the multicenter observational study TOOL [abstract no. PB0564]. Res Pract Thromb Haemost. 2021;5(Suppl 1):PB0564.

48. Horvais V, Drillaud N, Sigaud M, et al. Real-world use of FVIII in people with severe hemophilia A without inhibitor on prophylaxis, comparing switched to rFVIII-Fc versus non-switched patients: a multicentre, retrospective, non interventional, before-after study with the French nationwide claims database (MOTHIF-II) [abstract no. PB0501]. Res Pract Thromb Haemost. 2021;5(Suppl 1):PB0501.

49. Horvais V, Drillaud N, Sigaud M, et al. Real-world annual bleeding rate in people with severe hemophilia A without inhibitor on prophylaxis, comparing switched to rFVIII-Fc versus nonswitched patients: a multicentre, retrospective, non interventional, before-after study with the French nationwide claims database (MOTHIF-II) [abstract no. PB0577]. Res Pract Thromb Haemost. 2021;5(Suppl 1):PB0577.

50. Carcao M, Zunino L, Young NL, et al. Measuring the impact of changing from standard half-life (SHL) to extended halflife (EHL) FVIII prophylaxis on health-related quality of life (HRQoL) in boys with moderate/severe haemophilia A: lessons learned with the CHO-KLAT tool. Haemophilia. 2020;26(1):73-8.

51. Yan S, Maro GS, Desai V, et al. A real-world analysis of commonly prescribed FVIII products based on U.S. medical charts: consumption and bleeding outcomes in hemophilia A patients. $\mathrm{J}$ Manag Care Spec Pharm. 2020;26(10):1258-65.

52. Shrestha A, Su J, Li N, et al. Physical activity and bleeding outcomes among people with severe hemophilia on extended half-life or conventional recombinant factors. Res Pract Thromb Haemost. 2021;5(1):94-103.

53. Bocchinfuso $\mathrm{S}, \mathrm{O}$ 'Donovan $\mathrm{M}$, Singleton E, et al. Perioperative management of patients with severe haemophilia A with recombinant factor VIII FC fusion protein (rFVIIIFc) [abstract no. ABS157]. Haemophilia. 2021;27(Suppl 2):ABS157.

54. Lehtinen AE, Baghaei F, Astermark J, et al. Surgical outcomes in patients with severe haemophilia receiving $\mathrm{rFVIIIFc}$ and $\mathrm{rFIXFc}$ : real-world experience in the Nordic countries [abstract no. P056]. Haemophilia. 2020;26(Suppl 2):54-5.

55. Königs C, Ozelo MC, Dunn A, et al. Final results of PUPs A-LONG study: evaluating safety and efficacy of rFVIIIFc in previously untreated patients with hemophilia A [abstract no. OC 03.2]. Res Pract Thromb Haemost. 2021;4(Suppl 1).

56. US National Institutes of Health. ClinicalTrials.gov identifier NCT02234323. 2021.http://www.clinicaltrials.gov. Accessed 10 Sep 2021.

57. Kobayashi M, Nogami K, Park Y-D, et al. Evaluating the effectiveness of recombinant factor VIII Fc fusion protein ( $\mathrm{rFVIIIFc)}$ in adolescents and children with hemophilia $\mathrm{A}$ in the real world in Japan: interim analysis of the multicenter, observational $\mathrm{Fc}$ adolescent and children treatment study (FACTs) [abstract no. PB0578]. Res Pract Thromb Haemost. 2021;5(Suppl 1):PB0578.

58. Ebbert PT, Xavier F, Malec LM, et al. Observational study of recombinant factor VIII-Fc, rFVIIIFc, in hemophilia A. Thromb Res. 2020;195:51-4.

59. Nestorov I, Neelakantan S, Ludden TM, et al. Population pharmacokinetics of recombinant factor VIII Fc fusion protein. Clin Pharmacol Drug Dev. 2015;4(3):163-74.

60. Katragadda S, Neelakantan S, Diao L, et al. Population pharmacokinetic analysis of recombinant factor VIII Fc fusion protein in subjects with severe hemophilia A: expanded to include pediatric subjects. J Clin Pharmacol. 2021;61(7):889-900.

61. Bukkems LH, Heijdra JM, Mathias M, et al. A novel, enriched population pharmacokinetic model for recombinant factor VIIIFc fusion protein concentrate in hemophilia A patients. Thromb Haemost. 2020;120(05):747-57.

62. Merlin S, Follenzi A. Escape or fight: inhibitors in hemophilia A. Front Immunol. 2020;11:476.

63. Malec L, Carcao M, Jain N, et al. RFVIIIfc for first-time immune tolerance induction (ITI) therapy: interim results from the global, prospective verITI-8 study [abstract no. OC 32.1]. Res Pract Thromb Haemost. 2021;3(Suppl 1).

64. Carcao M, Shapiro A, Hwang N, et al. Real-world data of immune tolerance induction using recombinant factor VIII Fc fusion protein in patients with severe haemophilia A with inhibitors at high risk for immune tolerance induction failure: a follow-up retrospective analysis. Haemophilia. 2021;27(1):19-25.

65. Klamroth R, Saleh M, Andersson H, et al. Interim data from a chart review study of patients with haemophilia A with inhibitors treated with recombinant factor VIII Fc fusion protein (rFVIIIfc) for immune tolerance induction [abstract no. ABS136]. Res Pract Thromb Haemost. 2021;5(Suppl 1):ABS136.

66. Königs C, Meeks S, Löfqvist M, et al. Final results of ReITIratea prospective study of rescue immune tolerance induction (ITI) with recombinant factor VIII Fc (rFVIIIFc) in patients who have failed previous ITI attempts [abstract no. PB0522]. Res Pract Thromb Haemost. 2021;5(Suppl 1):PB0522.

67. Rayment R, Chalmers E, Forsyth K, et al. Guidelines on the use of prophylactic factor replacement for children and adults with haemophilia A and B. Br J Haematol. 2020;190:684-95.

68. Iorio A, Krishnan S, Myrén KJ, et al. Indirect comparisons of efficacy and weekly factor consumption during continuous prophylaxis with recombinant factor VIII Fc fusion protein and conventional recombinant factor VIII products. Haemophilia. 2017;23(3):408-16.

69. Graf L, Yan S, Shen MC, et al. A systematic review evaluating the efficacy and factor consumption of long-acting recombinant factor VIII products for the prophylactic treatment of hemophilia A. J Med Econ. 2020;23(12):1493-8.

70. Hakimi Z, Santagostino E, Postma MJ, et al. Recombinant FVIIIFc versus BAY 94-9027 for treatment of patients with haemophilia A: comparative efficacy using a matching adjusted indirect comparison. Adv Ther. 2021;38(2):1263-74.

71. Bullement A, McMordie ST, Hatswell AJ, et al. Cost-effectiveness analysis of recombinant factor VIII Fc-fusion protein ( $\mathrm{rFVIIIFc)}$ for the treatment of severe hemophilia A in Italy incorporating real-world dosing and joint health data. PharmacoEconomics Open. 2020;4(1):133-42.

72. Bullement A, Knowles ES, DasMahapatra P, et al. Cost-effectiveness analysis of $\mathrm{rFVIIIFc}$ versus contemporary $\mathrm{rFVIII}$ treatments for patients with severe hemophilia A without inhibitors in the United States. Pharmacoeconomics-Open. 2021. https://doi.org/ 10.1007/s41669-021-00283-6. 